\title{
Bilingüismo: herramienta clave en el contexto universitario ${ }^{1}$
}

\author{
Carmen Beatriz Araujo Quiroz ${ }^{2}$ \\ Universidad Popular del Cesar - Colombia \\ carmenaraujo@unicesar.edu.co
}

Recepción: 17/06/2012

Evaluación: 15/09/2012

Aceptación: 03/11/2012

Artículo de Reflexión

DOI: http://dx.doi.org/ 10.9757/Rhela.20.09

\section{"Los límites de mi lengua son los límites de mi mundo" Ludwig Wittgenstein}

\section{RESUMEN}

Este estudio constituye una reflexión crítica con enfoque sociocultural sobre la necesidad de repensar el bilingüismo en la educación superior. Su propósito principal se orienta hacia la comprensión de la educación actual como una práctica multilingüe o plurilingüe, en el marco de la sociedad del conocimiento. Se estructuró desde la Universidad Popular del Cesar como caso para el análisis y se desarrolló desde la metodología cualitativa de la investigación social para comprender la forma en que los actores interpretan y construyen sus propios significados y situaciones dentro y fuera del aula. Se abordan aspectos claves del bilingüismo y multilingüismo, tales como la acción sociopolítica y socioeconómica y las relaciones laborales y formativas de las diferentes profesiones. Como resultado se concluye que el uso funcional de las lenguas extranjeras implica negociación de sentidos desde la relación lengua, cultura y pensamiento.

Palabras clave: Revista Historia de la Educación Latinoamericana, bilingüismo, multilingüismo, contexto universitario, sociocultural.

1 Artículo de reflexión a partir del proyecto "Diseño e implementación de un módulo orientado hacia el desarrollo de habilidades comunicativas de la lengua inglesa en hablantes hispanos, con enfoque autónomo como estrategia pedagógica". Código: 022 2008. Vicerrectoría de Investigación-División de Investigación. Universidad Popular del Cesar.

2 Licenciada en Filología e Idiomas de la Universidad Libre de Colombia, Especialista en Pedagogía para el Aprendizaje y Desarrollo Autónomo, Doctorante en Ciencias de la Educación de la Universidad de Caldas de Manizales-RUDECOLOMBIA, Docente investigadora Universidad Popular del Cesar, Valledupar, Cesar Coordinadora del grupo de investigación GIELEHLA. 
Bilinguialism: a key tool in the university context

"The limits of my language are the limits of my world"

Ludwig Wittgenstein

\section{Bilinguismo: ferramenta chave no contexto universitário}

"Os limites de minha língua são os limites de meu mundo" Ludwig Wittgenstein

\section{RESUMO}

Este estudo consiste numa reflexão crítica com enfoque sociocultural sobre a necessidade de repensar o bilinguismo na educação superior. Seu propósito principal se orienta a partir da compreensão da educação atual como uma prática multilíngue ou plurilíngue no marco da sociedade do conhecimento. Estruturouse a partir da Universidade Popular de Cesar como caso para a análise e se desenvolveu com a metodologia qualitativa da pesquisa social, para compreender a forma em que os atores interpretam e constroem seus próprios significados e situações dentro e fora da aula. São abordados aspectos chave do bilinguismo e multilinguismo tais como a ação sociopolítica e socioeconômica e as relações laborais e formativas das diferentes profissões. Como resultado, conclui que o uso funcional das línguas estrangeiras implica negociação de sentidos a partir da relação língua, cultura e pensamento.

Palavras-chave: Revista História da Educação Latinoamericana, bilinguismo, multilinguismo, contexto universitário, sociocultural.

\section{INTRODUCCIÓN}

El bilingüismo y el multilingüismo coinciden en las esferas universitarias, en las diferentes áreas y disciplinas, como herramienta para la negociación de sentidos en la intercomunicación. Las diferentes lenguas convergen ahí como una vehiculización del conocimiento científico y tecnológico, propio de la formación profesional del siglo XXI. La sociedad exige a la universidad, desde los nuevos retos de la educación, la formación de un sujeto con responsabilidad social, sensibilidad cultural y respeto por la diversidad. El presente artículo se constituye en una reflexión crítica sobre algunos aspectos importantes del bilingüismo y multilingüismo en el contexto universitario. 
Para orientar la postura se plantea el siguiente cuestionamiento: ¿subyace el bilingüismo en el contexto universitario como componente transversal para la negociación de sentidos y contribuye al desarrollo integral en la educación superior?

El anterior interrogante surge en el marco del proyecto de investigación titulado Diseño e implementación de un módulo orientado hacia el desarrollo de habilidades comunicativas de la lengua inglesa en hablantes hispanos, con enfoque autónomo como estrategia pedagógica; en el que se toma como muestra los dieciocho programas académicos de pregrado correspondientes al calendario académico 2009-II y 2010-I de la Universidad Popular del Cesar.

Para el desarrollo de la temática se tuvo en cuenta las relaciones del bilingüismo con la formación universitaria, las necesidades del contexto social, entre otros aspectos, para concluir con una reflexión sobre la necesidad de repensar el bilingüismo y planificar la enseñanza de lenguas desde sus aspectos sociales e instrumentales, aludiendo al sentido de que toda experiencia educativa es una experiencia lingüística.

\section{El Bilingüismo}

En la consulta sobre el bilingüismo se han encontrado varios énfasis; algunos de ellos enfocados hacia cuestiones de índole lingüística y sus materiales educativos apoyan la enseñanza de la lengua extranjera desde aspectos estructurales de esta, como lo son, la gramática, el léxico, el préstamo lingüístico, las formas, los códigos, las categorías de uso, variantes semánticas y pragmáticas discursivas.

Uno de estos énfasis es el de Zentella, ${ }^{3}$ el cual define el bilingüismo como la disposición que tienen las personas de manejar al menos dos lenguas distintas; las cuales son capaces de manipular y mezclar para alcanzar sus necesidades de discurso, y expresar su identidad multicultural. Otros énfasis se orientan por la vertiente de lo social, que a la vez encierra una visión más política. Según Royo esto obedece a que:

3 La autora advierte la diferencia entre crecer bilingüe y adoptar un bilingüismo tardío, como es el caso del ambiente universitario. Cecilia Zentella, Growing up bilingual (Boston, MA: Blackwell, 1997), 61-74. 
El bilingüismo es una actitud mental de respeto y de igualdad, a que ninguna lengua es superior ni prioritaria; en el bilingüismo, las dos lenguas tienen igualdad de consideración con independencia de su peso demográfico y económico; pero el bilingüismo además, comporta una práctica social de respeto y cortesía, unos hábitos que en parte aún hemos de adquirir y que será bueno que se vayan incorporando a la convivencia, a manejar las dos lenguas con respeto y aplicar algunos criterios orientadores. ${ }^{4}$

Por ello, estos elementos que reciben los profesionales en la formación integral deben construir una igualdad lingüística. Lo anterior se viabiliza a través de protocolos que establezcan las reglas de juego; brindando así la oportunidad al profesional de elegir una lengua de su comodidad, matizada a través del currículo y formalizada en el plan de estudios. El estatus de una lengua y las actitudes frente al bilingüismo son factores macro sociológicos que condicionan el desarrollo del mismo, ${ }^{5} \mathrm{El}$ bilingüismo en el marco de la educación superior se convierte en una herramienta clave que incide en la calidad del participante que se forma; debido al trasfondo social tanto de la lengua de sus enseñantes como la de los aprendientes.

En contraste con lo anterior, el bilingüismo es visto como elemento de exclusión social para quienes no han tenido acceso a una formación lingüística de calidad; algunos defienden y valoran el bilingüismo como fenómeno enriquecedor y propiciador de hermandad entre los pueblos, ${ }^{6}$ mientras que otros lo consideran un elemento atrofiador de lenguas y culturas, instrumento de dominación, subordinación y colonialismo de las castas euroamericanas, ${ }^{7}$ ostentadoras del poderío económico y, por qué no decirlo, productoras de conocimiento científico y tecnológico.

En consecuencia, es válido considerar el bilingüismo como un área de trabajo asociada a todas las demás por su valor en el manejo de la información y su transversalidad en todos los saberes. Además, desde las esferas sociopolíticas se le atribuye un valor especial como componente del desarrollo de

4 Jesús Royo, Argumentos para el Bilingüismo (España: Novagrafik,2000),171

5 Bárbara Abdelilah, El desafío del bilingüismo: Crecer y vivir hablando varios idiomas (Madrid: España. Ediciones Morata, 2007), 28.

6 Por su parte, la corriente de las naciones unidas aduce a valores de humanismo como proceso en el que está inmerso el multilingüismo. Conferencia General de la Unesco, Declaración universal de la diversidad cultural para el diálogo y el desarrollo, 2003. Serie sobre la diversidad cultural No.1 http://unesdoc.unesco.org/images/0012/001271/127162s.pdf. (octubre 82010 ).

7 Algunos autores advierten los albores de la tercera colonización del mundo para los países en desarrollo. Donaldo Macedo, Bessie Dendrinos y Panayota Gounaris, Lengua, Ideología y Poder: la hegemonía del inglés (Barcelona: Editorial GRAO, de IRIF S.L.C:/ Francese Terraga, 2005), 21. 
una comunidad y como un eje que permea la calidad del sistema educativo. Por otra parte, una corriente del bilingüismo se asocia como elemento clave y estrategia de las castas elitistas, ${ }^{8}$ que lo ponen de bandera y estandarte de su publicidad.

Desde una perspectiva europea, el dominio de lenguas significa "la libertad de circulación y se considera la primera de las actitudes más útiles para encontrar un buen empleo". ${ }^{9}$ Las lenguas extranjeras son consideradas parte de las competencias de base y su dominio ha pasado a ser uno de los indicadores de la calidad de la educación. Por lo tanto, no se concibe un profesional monolingüe en un sistema educativo del siglo XXI en el que es evidente la necesidad de una apertura a las lenguas y a las culturas.

En Colombia lo anterior se hace evidente en el Programa Nacional de Bilingüismo ${ }^{10}$ al decidirse por el idioma inglés como objeto del Bilingüismo Nacional, cuando en la Ley General ${ }^{11}$ de Educación la enseñanza y aprendizaje de lenguas extranjeras no específica cuál de ellas se debe impartir; igualmente, la mencionada Ley dedica algunos de sus articulados a las demás lenguas nacionales advirtiendo sobre la etnoeducación dado que Colombia es una nación plurilingüe.

En el panorama nacional es ampliamente conocido el nivel deficiente en la lengua extranjera inglés y las dificultades en el dominio de la lengua materna (castellano) con que ingresan de la media escolar a la educación superior, estas deficiencias persisten a lo largo del sistema educativo Colombiano, en el que tampoco se percibe un domino de la lengua materna. ${ }^{12}$ En el caso particular de la Universidad Popular del Cesar, ${ }^{13}$ en la enseñanza de lenguas extranjeras, el inglés presenta el 93,2\% de preferencia y el 6,8\% se orienta por el idioma francés; las demás lenguas actualmente no hacen presencia en

8 El bilingüismo es una estrategia publicitaria para enganchar clientes y vender servicios de la educación superior, generando sentimientos de impotencia por parte de aquellos que no pueden acceder a estos supuestos servicios de calidad. Sivie Didou, Internacionalización y proveedores externos de la educación superior en América Latina y el Caribe (México: Colección biblioteca de la Educación Superior serie investigaciones. Iesalc Unesco, 2005), 26.

9 La perspectiva actual mundial enfatiza la hegemonía del inglés, aunque, en la tendencia de la comunidad europea se agruparon las lenguas por su cercanía y relatividad en categorías. Andrés González y Luis Mari Gómez La Enseñanza de las Lenguas Extranjeras desde una Perspectiva Europea (España: Ministerio de Educación y Ciencia. Omagraf, S.L. 2005), 12,15.

10 Colombia,Ministerio de Educación Nacional. Programa Nacional de Bilingüismo Colombia 2004-2019. Inglés como Lengua Extranjera: una estrategia para la competitividad.

11 Colombia. Ministerio de Educación Nacional Ley 115. Artículo 1. (Bogotá: Editorial Universidad Nacional. 1994).

12 Instituto Colombiano para el fomento de la Educación Superior. Informe de resultados pruebas SABER 11 y SABER PRO. Serie 11 (ICFES, Colombia, 2011).

13 Universidad Popular del Cesar. Informe estadístico de matrículas por estrato socioeconómico. Serie 2011-1. (Centro Administrativo de Registro y Control Académico. CARCA 2011). 
los demás planes de estudios y servicios ofertados por la Institución. Por su parte, el panorama de la lengua materna castellano se limita al plan de estudio de la Licenciatura en lengua castellana e inglés.

El contexto universitario por su finalidad se convierte en el espacio propicio para analizar que la hegemonía lingüística no solo se contempla en la participación de la enseñanza de una sola lengua y cultura extranjera, en este caso el inglés. De ahí que, el bilingüismo lengua materna-inglés esté presente en todas aquellas instituciones de educación superior donde el inglés no es la lengua materna de esa región, desfavoreciendo otras lenguas y culturas.

Por lo anterior, es válido considerar que en la educación superior el término de bilingüismo necesita resignificar su esencia, y su enfoque hacia un contexto más inclusivo respecto de la diversidad lingüística y cultural de la humanidad que reclama las bases de un plurilingüismo en el marco de una educación integral. De allí que, el modelo actual de educación requiere cerrar las brechas de un tejido social en el cual los aspectos cognitivos, lingüísticos, culturales, sociales, políticos y económicos sean materia de análisis al momento de la planeación y orientación de la práctica educativa, aprovechando los beneficios y previendo los peligros que encierra el bilingüismo desde lo sociocultural.

\section{El Bilingüismo en el proceso de formación universitaria}

La presente reflexión se dará a la luz de los postulados de Siguan y Mackey ${ }^{14}$ quienes consideran que los programas bilingües requieren una planificación de la enseñanza de ambas lenguas, una de las cuales suele ser la lengua materna de los alumnos. Por ello, tanto la lengua materna como la lengua extranjera requieren ser usadas como lenguas vehiculares de comunicación e instrucción en algunas asignaturas. En el ámbito de la educación superior conviene precisar el sentido educativo y formativo que tiene el bilingüismo con las relaciones entre los componentes profesor-estudiante contexto socialcurrículo y universidad-sociedad-conocimiento.

14 Estos autores consideran que en el marco del proceso enseñanza aprendizaje de las lenguas, estas se relacionan actuando como vehículo de comunicación y como instrucción. Miquel Siguán, y William F. Mackey, Éducation et bilinguisme (París: UNESCO Delachaux et Niestlé, 1986). 
Con respecto a la relación profesor-estudiante, en el marco de la educación superior, el bilingüismo desempeña un papel importante, ya que por un lado aporta a factores individuales (actitudinales y aptitudinales) y por otro a factores contextuales (adaptarse a las realidades y necesidades sociales). Además, la enseñanza bilingüe ha favorecido el desarrollo individual del estudiante porque facilita los procesos de cognición, y comunicación en la interacción social y cultural a partir del aprendizaje tanto de las lenguas en sí como de otros contenidos.

Lo anterior implica un proceso de doble vía (lo educativo y lo social) para el profesor cuyas necesidades de renovación pedagógica están marcadas por desarrollo de otros campos de la ciencia y la tecnología que desde un mercado globalizado permean las relaciones humanas, dado que la enseñanza en general es una red de relaciones que se teje lingüísticamente y que en el momento actual está mediada tecnológicamente. Así pues, esta doble relación propende por un cambio de actitud que debe reflejarse en los asuntos pedagógicos, didácticos, metodológicos, en los mediadores y por supuesto en el currículo.

En ese orden de ideas, el contexto social se refleja en el currículo de las instituciones de educación superior, paralelo a los procesos de aprendizaje de lenguas desarrollan otras actividades de formación que incluyen subprocesos relacionados con aprendizaje y adquisición de lenguas extranjeras, afinación de habilidades de la lengua materna, desarrollo de habilidades técnicas e instrumentales para el manejo de información útil y pertinente con su formación profesional. En estos subprocesos, lo cognitivo e instrumental constituye la funcionalidad del bilingüismo con relación a la apropiación, uso y producción de conocimiento. ${ }^{15}$

En cuanto a los procesos académicos, el bilingüismo está presente en el desarrollo intelectual y en los subprocesos que tienen que ver con las competencias metacognitivas. ${ }^{16}$ También con los subprocesos de las competencias

15 En el Artículo 6, literal b de la Ley 30 de1992 se plantea "Trabajar por la creación, el desarrollo y la transmisión del conocimiento en todas sus formas y expresiones y, promover su utilización en todos los campos para solucionar las necesidades del país". Colombia, Congreso de la República, Ley 30: Fundamentos de la Educación Superior: Capítulo I, Artículo 5. (Bogotá, Diario Oficial No. 40.700, de 29 de diciembre de 1992).

16 Competencia cognitiva en la educación superior. las competencias metacognitivas relacionadas son la planeación y control del acto educativo, consistente en reflexionar cómo uno mismo aprende, crea, razona, decide, actúa y siente. María Sanz, Competencia cognitiva en la educación superior (Madrid: España, NARCEA, S.A, 2010):103. 
sociales ${ }^{17}$ las cuales exigen altos niveles de eficiencia y comprensión en la comunicación para el contexto universitario donde en primera instancia se deben alcanzar los estándares de comunicación en la lengua materna, para luego ser transferidos a las lenguas extranjeras. ${ }^{18}$

El bilingüismo, en los subprocesos instrumentales apoya el desarrollo de las habilidades técnicas y metodológicas, ayuda a la construcción del conocimiento y a la resolución de problemas. Interpretando a Velázquez, ${ }^{19}$ el bilingüismo está enfocado, primeramente, a la adquisición de una segunda lengua (L2) de prestigio (inglés, francés, alemán, etc.) y a mantener, e incluso a mejorar, el conocimiento de la primera lengua (L1).

Lo que se persigue mediante el desarrollo de la educación bilingüe, y por consiguiente del bilingüismo, es la formación de individuos capaces de lidiar con situaciones en las que se requiera hacer intercambios comunicativos mediante estas lenguas, ya sea de negocios, turismo, tecnológicos y de estudio. La ventaja de este tipo de educación bilingüe (instrumental) señala que en ningún momento se persigue la eliminación de la lengua materna; en este caso el español, por el contrario permite reflexionar sobre el uso y funcionalidad de la lengua materna.

En el contexto de la educación superior, el bilingüismo desarrolla procesos curriculares que requieren estar en consonancia con las tendencias globales, por ello el nivel superior se articula en comunidades académicas con entidades homólogas en el ámbito internacional ${ }^{20}$ en el que las lenguas constituyen el vehículo de comunicación y no es posible desarrollarlo sin el dominio eficaz de las competencias comunicativas y socioculturales, lo que incluye necesariamente lenguas y culturas extranjeras.

Los currículos de los programas universitarios necesitan formar diversas clases de profesionales bilingües con diferentes énfasis y enfoques de acuerdo

17 Estas competencias se refieren al conjunto de habilidades que le permiten al individuo la articulación armónica al grupo social. Sanz, "Competencia", 63.

18 La transferencia en el marco de la enseñanza aprendizaje de lenguas se refiere a la aprensión de elementos lingüísticos, sociales y culturales de ambas lenguas, que luego son representados a través de la interacción en la comunicación. Sonia White Soltero, "Dual Language: Teaching and Learning in two Languages," (Boston, MA: Allyn y Bacon, 2004), citado por Francisco Ramos, "Programas bilingües y formación de profesores en Andalucía," Revista Iberoamericana de Educación No. 44 (2007,): 133,146.

19 María Velázquez, Breve Introducción al Fenómeno del Bilingüismo en México. (México: Universidad de Guadalajara -Sincronía Primavera ,2005). http://www sincronia.cucsh.udg.mx/velazqueza05

20 República de Colombia Ley 30. Congreso de la República. Capítulo II, Artículo 6. Literal h. (Bogotá, Diario Oficial No. 40.700, de 29 de diciembre de 1992). 
a sus necesidades en la vida profesional. Por ello, se requiere como primera medida que el bilingüismo centre su atención en currículos pertinentes para la formación de maestros de lengua materna y extranjeras con el objeto de promover profesionales con verdaderos niveles académicos y lingüísticos. ${ }^{21}$ Como segunda medida, los currículos de formación de profesionales con énfasis en contextos internacionales. Este tipo de profesionales requieren de currículos contextualizados con las dinámicas propias de la globalización, de la economía y de la sociedad en su conjunto. En tercera instancia, los currículos orientados a la formación de profesionales con énfasis en contextos locales. Este prototipo de profesionales requiere de currículos orientados principalmente a las dinámicas de la región, las cuales dependen fuertemente de las habilidades de la lengua materna.

El sentido educativo y formativo que tiene el bilingüismo en el contexto universitario precisa una relación dentro y fuera del contexto de formación profesional. El bilingüismo permite al profesional vivir una relación de sincronía con el desarrollo epistemológico del conocimiento que le posibilita un proceso de actualización continua.

El medio laboral y profesional en el que se desempeña un individuo pone de manifiesto la relación universidad-sociedad-conocimiento. Allí, el bilingüismo subyace en los subprocesos que tienen que ver con la construcción de la identidad, de la imagen y la autoimagen, el estatus y el prestigio; no solo de la institución, sino también de los participantes. Bajo esta perspectiva, fuera de los muros y lejos de los profesores, los profesionales dependen de las capacidades adquiridas para continuar su aprendizaje basado en el conocimiento previo y las nuevas experiencias. Capital sobre el cual soportan su accionar político social y económico en una compleja red de relaciones de diversa naturaleza.

Lo anterior permite reflexionar sobre el compromiso social de la educación superior, su necesidad de construir un currículo contextualizado hacia entornos bilingües, multilingües y multiculturales. Este compromiso le exige además la formación de profesionales competentes para enfrentar las tareas del desarrollo y avance de los pueblos, con suficiente solidez para responder a los espacios de transición propios de la globalización. Por lo que, es necesario

21 El currículo pertinente se gesta en el seno mismo de la sociedad, nace de las necesidades de los participantes Nancy Cloud, Fred Genesee, y Else Hamayan, Dual language instruction: A handbook for enriched education. (Boston, MA: Heinle y Heinle. 2000). 
tener bien claro las luchas de los pueblos por sus identidades, su cultura, su sistema de pensamiento, su accionar, el reconocimiento y garantía de sus productos científicos y tecnológicos independientemente de su ubicación geográfica, estado de desarrollo y nivel socioeconómico.

\section{Componente socio-político y las relaciones universidad sociedad}

Desde el papel del bilingüismo y el multilingüismo en la comunicación y los ambientes para la ciencia y la tecnología se perciben otras connotaciones adheridas a la lengua, referentes al modo en que esta afecta los sentimientos personales y la identificación colectiva; afecta al poder y a la economía; como también, a los derechos civiles de los individuos y sus expectativas de progreso y de integración en la sociedad..$^{22}$ Cuando estos individuos egresan de una Institución de Educación Superior, la integración a la sociedad les demanda altos niveles de competitividad relacionados con su articulación al mundo laboral.

Desde los aspectos sociales y políticos, la lengua es un elemento fundante en la construcción de las naciones, de sus identidades nacionales y culturales en tiempos de globalización..$^{23}$ Fishman, citado por Baker, plantea que la lengua es un índice de la cultura. Una lengua que ha estado históricamente asociada a una cultura expresa mejor, con mayor facilidad y mayor riqueza los elementos de dicha cultura. Por ello, factores como el prolongado dominio económico, político y cultural aceleran la asimilación lingüística y la desaparición de las lenguas y culturas minoritarias, asimilando un proceso casi imperceptible de colonización que debilita los límites entre las lenguas y por ende su prestigio.

Partiendo que la lengua es un índice de la cultura, existen razones políticas, literarias y científicas que son útiles para argumentar que el aprendizaje de un idioma extranjero es una experiencia enriquecedora cultural e intelectualmente. Desde una perspectiva reducida, el aprendizaje de una lengua extranjera puede ser instrumento de devastación de la cultura local de los pueblos. ${ }^{24}$ Es claro pensar que corresponde a la misión social de la universi-

22 Otra corriente aduce a valores de civilidad y considera al bilingüismo como herramienta que permite la integración. Didou, "Internacionalización y proveedores," 210.

23 Macedo, Dendrinos y Gounaris, Lengua, Ideología y Poder, 24.

$24 \mathrm{El}$ aprendizaje de una lengua extranjera constituye un acercamiento a la cultura, historia, modos de vida, entre otros, que de hecho han influenciado a muchas personas que optan por los modos del otro restando valor a lo propio. Soraya Sánchez, "La 
dad, hacer la defensa de la lengua y la cultura de los pueblos. Esto obedece a su responsabilidad con la sociedad y ha de formar parte de su política educativa y lingüística.

Lo precedente invita a pensar el bilingüismo como un fenómeno de doble vía; en el que dos lenguas están presentes en un mismo territorio, momento y contexto. De una parte, se presenta la lengua materna, la producción de esta, la cultura y las ideas de aquellas comunidades que la hablan y, por otra parte, el bilingüismo como fenómeno representa el papel de reproducción de las ideas, cultura y pensamiento de los pueblos en otras lenguas diferentes a la propia cuyos hablantes nativos y foráneos las están usando como vehículo de las ideas expresadas en ellas.

En consecuencia, las negociaciones de sentido están predeterminadas por las relaciones previas del participante construidas a través de su lengua y cultura materna y su conocimiento intelectual en lo académico y el reconocimiento de la propia identidad. ${ }^{25}$ Esta identidad se debe cristalizar en la adquisición y desarrollo de la conciencia lingüística, entendida desde el valor social que representa cada pueblo y grupo en la diversidad cultural de la raza humana.

\section{Componente interdisciplinario del bilingüismo}

La educación superior es un contexto multilingüe permeado por diversos componentes tales como calidad, eficiencia, productividad, competitividad y responsabilidad social, como componente que exige el siglo XXI para una educación de calidad. En este campo, el bilingüismo subyace como uno de esos componentes que inciden en el momento de la interpretación, a partir de referentes o mediadores dados, desde o en una lengua extranjera.

En la educación superior se hace evidente que el bilingüismo es una condición de calidad porque el trabajo académico ostenta una visión de universalidad del conocimiento producido a través de la investigación; reproducido desde la docencia, recreado contextualizado y difundido en las universidades a través de múltiples formas. Este conocimiento está configurado como

construcción social de dificultades de aprendizaje en las prácticas educativas", Revista Brasilera Educación especial. Marilia, SetDez Volumen 14, № 3 (2008): 364.

25 Sánchez, La construcción social, 348. 
una cultura propia de la academia; por ende, desde allí la formación de los profesionales, especialmente a nivel de maestría y doctorado, requiere el desarrollo de una capacidad para insertarse en la ciencia global y formar parte activa en redes de investigación.

Igualmente, permite interactuar con colegas de diferentes partes del mundo en sus diferentes lenguas y culturas, propiciando la movilidad internacional de investigadores, profesores y estudiantes, la doble titulación y la homologación de títulos. ${ }^{26}$

Por otra parte, la tendencia y exigencia del presente siglo está llevando a la universidad a la acreditación e internacionalización de programas académicos e instituciones, armonizando los criterios de calidad desde equipos de pares internacionales de alto nivel que han de homogeneizar la educación en el marco de la sociedad mundial, las ciencias y las redes.

En el contexto universitario el bilingüismo incide en la interpretación, apropiación negociación y transferencia de los significados y sentidos entre áreas lingüísticas y áreas no lingüísticas; en los temarios científicos el bilingüismo subyace como uno de esos componentes. Cuando se habla de negociación de sentidos en el escenario de la Universidad, se contempla la relación de lengua-ciencia-conciencia-cultura y se pone en manifiesto el esquema de pensamiento construido del aprendiente a través del uso de mediadores diseñados en lenguas diferentes por escritores de culturas diferentes.

\section{Necesidad de repensar el bilingüismo en el contexto universitario}

Repensar el bilingüismo en la educación superior dentro del marco de la globalización implica trabajar la pluriculturalidad que impone a todos el desafío de conservar la diferencia frente a la homogenización como la impronta del tercer milenio. Hace falta retomar el pensamiento crítico-analítico que pueda plantear alternativas para el desarrollo de la humanidad y el de los sujetos mismos; ya que en el seno de la sociedad global actual, la pertenencia a una institución latinoamericana conduce a una concesión a priori en la que somos considerados de manera prejuiciada y obligados a aprender la lengua de los imperios económicos.

26 Apuntando a los planteamientos curriculares desde la implicación social del bilingüismo y la concepción de la calidad de la educación superior, entendida el bilingüismo como un valor agregado para la homologación y validez del conocimiento en otras latitudes. Diana Lagos, Internacionalización del curriculo una condición de calidad en la educación superior. (Colombia: Consejo Nacional de Acreditación-CNA ,2009). 
Lo anterior obedece a que prima la necesidad de una transformación de la de Educación Superior a la luz del ethos cultural, ${ }^{27}$ el cual permitiría a los diferentes programas académicos que confluyen en el marco de las universidades superar el positivismo absoluto con una mentalidad reflexiva; lo cual conlleva a encontrar la razón en la práctica, en la orientación, la responsabilidad de vivir, el sentido de la vida, la sociedad y la historia, la experiencia del cuerpo como fundamento de toda educación y a la expresión del lenguaje como la huella de estar el hombre en el mundo, su tradición fundida opacada por el marcado etnocentrismo euroamericano, que no permite asumir una posición autocrítica que refleje el desarrollo de competencias relacionadas a la comprensión y ampliación de conocimientos socioculturales con relación a otras latitudes y a otras disciplinas en el contexto de educación superior.

Las universidades, en su papel de generadora de cultura y conocimientos, comparten a lo largo y ancho del planeta su fuerte deseo de aprender unas de otras, de mostrar, compartir e intercambiar lo mejor del producto de su práctica. Esta nueva estrategia de diálogo se enlaza desde el multilingüismo como base de cooperación estimulante y retadora para la negociación de sentidos entre ciencia, lengua y cultura. Esta trilogía ha alcanzado el estatus de sociedad del conocimiento, donde unos validan o invalidan a otros en una espiral infinita en la que se entrelazan lengua-cultura-conocimiento en un solo escenario llamado universidad; donde se borran los límites impuestos por la geografía y la lengua, pero se han construido fuertes y grandes brechas de prejuicios y subvaloraciones enraizadas en estereotipos de visión de sociedad, que como lo refiere Amin Maalof constituyen su bien nominado término de identidades asesinas, y en términos de Chimamanda Adichie encierran "The danger of a single story". ${ }^{2}$

En el seno de la sociedad global la pertenencia a una institución latinoamericana conduce a una concesión a priori en la que somos considerados de manera prejuiciada y obligados a aprender la lengua de los imperios económicos. Por ello conviene que el bilingüismo sea materia de gran preocupa-

27 El ethos cultural es visto como la identidad, el modo de ser y de vivir de una comunidad en particular, en la que prevalecen una serie de valores culturales, que controlan o determinan las praxis sociales. Martina Tuts, "Las lenguas como elementos de cohesión social. Del multilingüismo al desarrollo de habilidades para la comunicación intercultural”, Revista de Educación, No. 343 (2007): 35-54.

28 Escritura africana que a través de su vivencia personal señala que la conciencia crítica cultural y la concepción de la diversidad étnica, son aspectos que no se tienen en cuenta en el momento de enseñar y advierte a los lectores padres y maestros del peligro que encierra obtener una sola mirada de la historia y de los países. Adichie, Chimamanda, The danger of a single story. (Oxford: Reino Unido, 2009).http://.www.ted.com/.../chimamanda_adichie_the_danger_of_a_single_story (agosto 17 2010) 
ción para la comunidad académica, debido a que valoriza temáticas de las diferentes disciplinas científicas como fuente de ideas; cuyo sustento teórico proviene de estudios e investigaciones realizadas por comunidades de investigadores de los diferentes escenarios universitarios mundiales que en su conjunto aportan a la construcción del conocimiento como práctica de la humanidad, independientemente de la lengua en que esté vehiculizado.

\section{CONCLUSIÓN}

El conocimiento en la práctica educativa es un bien migratorio no solo entre países y continentes y entre las diferentes disciplinas, sino también, entre las diferentes lenguas y culturas; lo que invalidaría el concepto de bilingüismo y validaría el de multilingüismo como pieza clave para la articulación de los componentes de la universidad como institución del siglo XXI, que universaliza las prácticas educativas como prácticas multilingües o plurilingües y no solo se enmarca en un bilingüismo sustrativo una de las lenguas está desvalorizada en el que el inglés ejerce el efecto de puente entre todas las culturas constituyéndose en la lengua franca si se vale el término de la sociedad del conocimiento. Entendiéndose que la geografía cultural encierra una naturaleza política en la que es importante considerar las lenguas y reconocer el papel de la diversidad lingüística para preservar en las sociedades derechos inalienables de justicia social en la que el territorio o la territorialidad como producto histórico funde las trazas de identidad de un pueblo.

\section{FUENTES}

Center for Applied Linguistics. Directory of Two-Way Bilingual Immersion Tables: Number of Districts and Schools by State. California: Center for Applied Linguistics, 2007. http: / /www. cal.org/twi / directory / index.html (agosto 27 2010)

Consejería de Educación. Plan de Fomento del Plurilingüismo: hacia un nuevo modelo metodológico. Andalucía España: Junta de Andalucía, 2005. http: / / www.juntadeandalucia. es (septiembre 9 2010)

Freeman, Yvonne; David, Freeman, y Sandra Mercury. Dual Language Essentials for Teachers and Administrators. Portsmouth, NH: Heinemann, 2005.

Gabinete de Prensa Cándida Martínez presenta en Bruselas el Plan de Fomento del Plurilingüismo a representantes de la Unión Europea, Junta de Andalucía, 2006. http:/ / www.juntadeandalucia.es1 (septiembre 15 2010)

García, Eugene. Teaching and Learning in Two Languages: Bilingualism and Schooling in the United States. Nueva York: Teachers College Press, 2005. 
Genesee, Fred. Program Alternatives for Linguistically Diverse Students. Santa Cruz, California: Center for Research on Education, Diversity y Excellence, 1999.

Instituto Colombiano para el Fomento de la Educación Superior. Resultados pruebas, SABER 11, SABER PRO, Colombia: ICFES, 2011.

Krashen, Stephen. Under Attack: The Case Against Bilingual Education. Culver City, CA: Language Education Associates, 1996.

Lasagabaster, David. "Bilingualism, Immersion Programmes and Language Learning in the Basque Country", Journal of Multilingual \& Multicultural Development, Vol.: 22 No. 5 (2001): 421

Lessow-Hurley, Judith. "The Foundations of Dual Language Instruction". Nueva York: Longman. 3. ${ }^{\text {e ed., } 2000 .}$

Lindholm-Leary, Kathryn. Dual Language Education. Clevedon,England: Multilingual Matters. 2001.

Ministerio de Educación, Cultura y Deportes, Marco común europeo de referencia para las lenguas: aprendizaje, enseñanza, evaluación. Madrid: Subdirección General de Cooperación Internacional, 2002.

Opeid, Universidad Popular Del Cesar. Informe estadístico de matrículas por estrato socioeconómico. Colombia, 2010.

Ramos, Francisco. "Programas bilingües en Estados Unidos y España: dos innovaciones en la enseñanza de idiomas", en Actas del XL Congreso Internacional de la Asociación Europea de Profesores de Español, (2006): 334-342.

República de Colombia. Ley General de la Educación. Ley 115. Colombia Universidad Nacional, 1994.

República de Colombia. Fundamentos de la Educación Superior. Ley 30 de Diciembre 28. Colombia Diario Oficial No. 40.700. 1992.

\section{REFERENCIAS}

Abdelilah, Bárbara. El desafío del bilingüismo: Crecer y vivir hablando varios idiomas. Madrid: España. Ediciones Morata, 2007.

Castro, Feinberg. Bilingual Education: A reference handbook. Santa Bárbara, CA: ABC-CLIO, Inc., 2002.

Cloud, Nancy, Genesee, Fred. y Hamayan, Elese. Dual language instruction: A handbook for enriched education. Boston, MA: Heinle y Heinle, 2000.

Conferencia General de la Organización de las Naciones Unidas para la Educación, Declaración universal de la diversidad cultural para el diálogo y el desarrollo, Paris: 2003. Serie sobre la diversidad cultural No.1 http://unesdoc.unesco.org/images/0012/001271/127162s.pdf. (Octubre 8 2010)

Didou, Sivie. Internacionalización y proveedores externos de la educación superior en América Latina y el Caribe. México: Colección biblioteca de la Educación Superior serie investigaciones-IESALC-Unesco, 2005. 
González, Andrés y Luis Marigómez. La Enseñanza de las Lenguas Extranjeras desde una Perspectiva Europea. España: Ministerio de Educación y Ciencia. Omagraf, S.L, 2005.

Lagos, Diana. Conferencia Internacionalización del currículo una condición de calidad en la educación superior. Bogotá: Consejo Nacional de Acreditación-CNA, 2009.

Macedo Donaldo, Bessie Dendrinos y Panayota Gounaris. Lengua, Ideología y Poder: la hegemonía del inglés. Barcelona: Editorial GRAO, de IRIF S.L.C:/ Francese Terraga, 2005.

Ramos, Francisco. "Programas bilingües y formación de profesores en Andalucía”, Revista Iberoamericana de Educación, No. 44. (2007): 146.

Royo, Jesús. Argumentos para el Bilingüismo. España: Novagrafik, 2000.

Sánchez, Soraya. "La construcción social de dificultades de aprendizaje en las prácticas educativas". Revista Brasilera Educación especial. Marilia, Set-Dez, Vol.: 14, No. 3(2008): 348.

Sanz, María. Competencia cognitiva en la educación superior. España: NARCEA, S.A, 2010.

Siguán, Miquel y Mackey, William. F. Éducation et bilinguisme. París, Unesco: Lauzanna: Delachaux y Niestlé, 1986.

Soltero, Sonia White. Dual Language: Teaching and Learning in two Languages. Boston, MA: Allyn y Bacon, 2004.

Tuts, Martina. “Las lenguas como elementos de cohesión social, Del multilingüismo al desarrollo de habilidades para la comunicación intercultural". Revista de Educación, No. 343 (2007): 54

Velázquez, María. Breve Introducción al Fenómeno del Bilingüismo en México. Universidad de Guadalajara - Sincronía- Primavera, 2005. http://www.sincronia.cucsh.udg.mk/ velazqueza05

Zentella, Cecilia. Growing up bilingual. Boston, MA: Blackwell, 1997.

Araujo Quiroz, Carmen Beatriz. "Bilingüismo: herramienta clave en el contexto universitario". Revista Historia de la Educación Latinoamericana. Vol. 15 No, 20, (2013): 189 - 204. 